\section{Nota informativa de seguridad para público general: "Los peligros del mal uso de los teléfonos celulares"} Safety note for the general public "the dangers of misusing mobile telephones"

Drs. Lientur Taha, Christian Dauvergne e Ing. Biomédico Guillermo Avendaño

El Instituto de Salud Pública de Chile a través del Subdepartamento de Dispositivos Médicos de la Agencia Nacional de Medicamentos, ANAMED, en conjunto con el Comité de Expertos de Dispositivos Médicos ${ }^{1}$, informa sobre los efectos biológicos que pueden producir las emisiones de radiación electromagnética provenientes de teléfonos celulares.

En las últimas décadas se ha producido un crecimiento explosivo de la comunicación inalámbrica, particularmente el uso de la telefonía celular. Se ha señalado que los campos electromagnéticos de la telefonía celular penetran los tejidos expuestos que absorben la radiación de microondas, por lo que representarían una fuente potencialmente dañina para la salud humana.

La radiación electromagnética, se caracteriza por sus longitudes de onda o por su frecuencia, dando origen al "espectro electromagnético". Puede manifestarse de diversas maneras tales como calor, sonido, luz visible, Rayos X o Rayos gamma. Todas estas formas de onda interaccionan con los seres vivos, pero algunas pueden ser percibidas y otras no. Por ejemplo, los seres humanos perciben la luz visible pero no pueden percibir los rayos $\mathrm{X}$ que son emitidos por los equipos de radiología.

La ciencia ha establecido que algunas frecuencias muy elevadas, que son portadoras de muy alta energía y producen un fenómeno conocido como

${ }^{1}$ Comité de expertos conformado por Dr. Lientur Taha, Dr. Christian Dauvergne e Ing. Biomédico Guillermo Avendaño. "ionización", pueden ser perjudiciales para la salud. En esta zona encontramos los Rayos X y los Rayos gamma. En la Figura 1 se puede observar el espectro electromagnético.

\section{Efectos de las emisiones de teléfonos celulares (radiación no ionizante)}

Como se puede observar en la Figura 1, la radiación que procede de un teléfono celular es del tipo no-ionizante y se encuentra en el rango de las ondas de radiofrecuencia. Es muy distinta a la radiación ionizante de los rayos $\mathrm{X}$, los cuales son muy dañinos. El teléfono celular es más bien como un horno de microondas de muy baja potencia. A corto plazo, estas radiaciones son inofensivas, pero a largo plazo podrían ser distintas.

En los últimos años, a nivel internacional ha surgido la pregunta: ¿los teléfonos celulares podrían provocar cáncer cerebral? Es muy pronto para saberlo. Se considera que el período de latencia, o tiempo entre la exposición y el reconocimiento de un tumor, es de alrededor de 20 años o más. Algunas publicaciones señalan que el efecto de la radiación de un teléfono celular sobre el cerebro sería similar a lo que le sucede a los alimentos colocados en el microondas. En términos simples "cocinarlo". Otras publicaciones indican que los teléfonos celulares además de provocar desarrollo de tumores y cáncer, podrían producir una serie de otros efectos sobre la memoria, ya que los lóbulos temporales de la memoria están localizados precisamente en la zona donde las personas sujetamos nuestros teléfonos celulares. En teoría, los niños estarían en un riesgo más alto que los adultos, porque el cráneo de un niño es más delgado comparado con el cráneo de un adulto, permitiendo así que la radiación penetre más profundo. Por otra parte, como sus cabezas son más pequeñas que las de los adultos, presentan una exposición proporcionalmente mayor al campo de radiación de radiofrecuencia de los teléfonos celulares. Además, los niños actualmente tienen la posibilidad de acumular más años de exposición a los teléfonos 


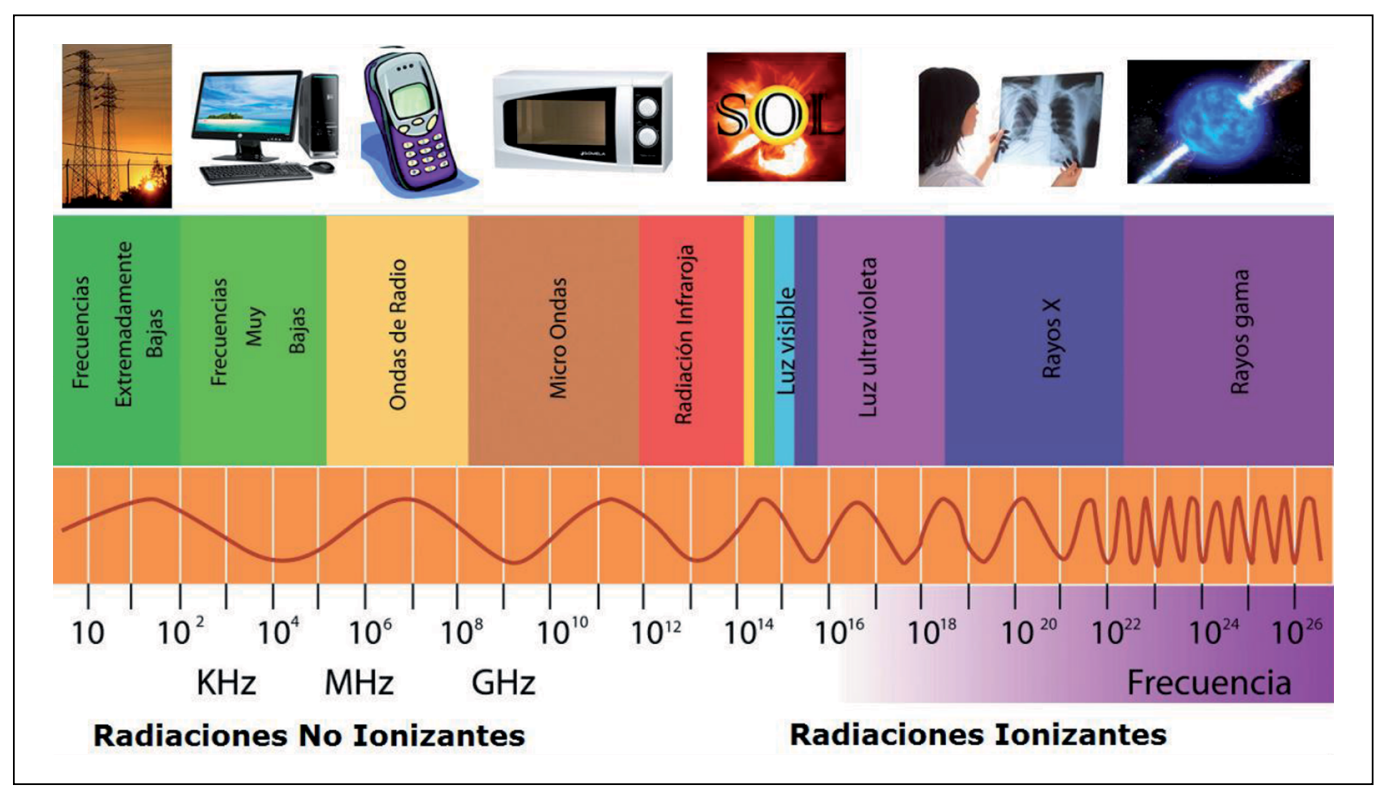

Figura 1.

celulares que los adultos. Consecuentemente el impacto de la radiación podría ser mucho más grande en los niños que en los adultos.

Por lo señalado, y considerando el gran aumento en el número de teléfonos celulares, a nivel científico ha surgido la necesidad de establecer los efectos biológicos que pueden provocar estas emisiones y las medidas correspondientes para evitar efectos dañinos para la salud.

Se han llevado a cabo varios tipos de estudios epidemiológicos para investigar la posibilidad de que exista una relación entre el uso de teléfonos celulares y el riesgo de tumores cerebrales cancerosos, tales como gliomas. Por lo general, los resultados de muchos estudios, no han proporcionado clara evidencia de una relación entre el uso de los teléfonos celulares y el cáncer; no obstante, ha habido hallazgos estadísticamente significativos en determinados subgrupos de personas. Algunos estudios señalan que el uso de los teléfonos celulares podría derivar en riesgo de padecer cáncer y provocar el efecto microondas ${ }^{2}$, producir enfermedad a los ojos (Cataratas), afectar las células e incluso atravesar

2"Efecto microonda": es una sintomatología específica propia de la exposición a bajos niveles de radiación, especialmente a las alteraciones neurológicas producidas por ondas moduladas por muy bajas frecuencias. los protectores celulares (barrera hematoencefálica). Además podría afectar la producción de hormonas relacionadas con el stress, entre otros.

La Agencia Internacional de Investigación de Cáncer, ha clasificado la Radiación Electromagnética producida por los teléfonos celulares como posiblemente carcinogénica para los seres humanos. $\mathrm{Y}$ a nivel mundial, actualmente se siguen realizando estudios para analizar más a fondo los posibles efectos a largo plazo del uso de teléfonos celulares ${ }^{3}$.

Se ha podido establecer que una persona que utiliza el teléfono celular a una distancia de entre 30 y 40 centímetros de su cuerpo, por ejemplo al escribir mensajes de texto o navegar por internet, estará mucho menos expuesta a campos de radiofrecuencia que quienes lo utilizan acercando el aparato a su cabeza ${ }^{4}$.

Considerando la mayor probabilidad de riesgo debido al uso cada vez más prolongado de los

\footnotetext{
${ }^{3}$ Nota de la Organización Mundial de la Salud OMS, "Campos electromagnéticos y salud pública: teléfonos móviles” (2011) link: http://www.who.int/mediacentre/ factsheets/fs193/es/

${ }^{4}$ Nota de la Organización Mundial de la Salud OMS, "Campos electromagnéticos y salud pública: teléfonos móviles” (2011) link: http://www.who.int/mediacentre/ factsheets/fs193/es/
} 
teléfonos celulares en las cercanías del cráneo, se ha estimado necesario difundir y recomendar un conjunto de medidas destinadas a proteger a la población de los riesgos producidos por el mal uso de los teléfonos celulares.

\section{Recomendaciones en el uso de teléfonos celulares}

1) Reducir el tiempo de las llamadas realizadas por teléfonos celulares.

2) Privilegiar el uso del altavoz, de "Manos Libres" y de "bluetooth".

3) Tener en consideración que los niños podrían ser más vulnerables a las radiaciones, por ende se aconseja a los padres educar a los hijos al respecto.

4) Usar el teléfono celular en forma perpendicular a la oreja.

\section{Referencias bibliográficas}

1. Zamorano M, Torres H, Soto E. Modelado y simulación del SAR debido a los campos electromagnéticos radiados por teléfonos celulares. Rev Fa Ing Universidad Tarapacá 2005; 13 (3): 114-21.

2. Nota de la Organización Mundial de la Salud OMS "Radiaciones ionizantes: efectos en la salud y medidas de protección" (2012) link: http://www. who.int/mediacentre/factsheets/fs371/es/

3. Volkow ND, Tomasi D, Wang GJ, et al. Effects of cell phone radiofrequency signal exposure on brain glucose metabolism. JAMA 2011; 305 (8): 808-13. [PubMed Abstract].

4. Hirose H, Suhara T, Kaji N, et al. Mobile phone base station radiation does not affect neoplastic transformation in BALB/3T3 cells. Bioelectromagnetics 2008; 29 (1): 55-64. [PubMed Abstract].

5. Oberto G, Rolfo K, Yu P, et al. Carcinogenicity study of $217 \mathrm{~Hz}$ pulsed $900 \mathrm{MHz}$ electromagnetic fields in Pim1 transgenic mice. Radiation Research 2007; 168 (3): 316-26. [PubMed Abstract].

6. Zook BC, Simmens SJ. The effects of pulsed 860 $\mathrm{MHz}$ radiofrequency radiation on the promotion of neurogenic tumors in rats. Radiation Research 2006; 165 (5): 608-15. [PubMed Abstract].
7. Ahlbom A, Green A, Kheifets L, et al. Epidemiology of health effects of radiofrequency exposure. Environmental Health Perspectives 2004; 112 (17): 1741-54. [PubMed Abstract].

8. Cardis E, Richardson L, Deltour I, et al. The INTERPHONE study: design, epidemiological methods, and description of the study population. European Journal of Epidemiology 2007; 22 (9): 647-64. [PubMed Abstract].

9. International Agency for Research on Cancer (2008). INTERPHONE Study: latest results update-8 October 2008. Lyon, France. Retrieved June $18,2012$.

10. The INTERPHONE Study Group. Brain tumour risk in relation to mobile telephone use: results of the INTERPHONE international case-control study. International Journal of Epidemiology 2010; 39 (3): 675-94. [PubMed Abstract].

11. Larjavaara S, Schüz J, Swerdlow A, et al. Location of gliomas in relation to mobile telephone use: a case-case and case-specular analysis. American Journal of Epidemiology 2011; 174 (1): 2-11. [PubMed Abstract].

12. Johansen C, Boice J Jr, McLaughlin J, Olsen J. Cellular telephones and cancer: a nationwide cohort study in Denmark. Journal of the National Cancer Institute 2001; 93 (3): 203-7. [PubMed Abstract].

13. Schüz J, Jacobsen R, Olsen JH, et al. Cellular telephone use and cancer risk: update of a nationwide Danish cohort. Journal of the National Cancer Institute 2006; 98 (23): 1707-13. [PubMed Abstract].

14. Frei P, Poulsen AH, Johansen C, et al. Use of mobile phones and risk of brain tumours: update of Danish cohort study. British Medical Journal 2011; 343: d6387. [PubMed Abstract].

15. Benson VS, Pirie K, Schüz J, et al. Mobile phone use and risk of brain neoplasms and other cancers: Prospective study. International Journal of Epidemiology 2013; First published online: May 8, 2013. doi:10.1093/ije/dyt072

16. Muscat JE, Malkin MG, Thompson S, et al. Handheld cellular telephone use and risk of brain cancer. JAMA 2000; 284 (23): 3001-7. [PubMed Abstract] 
17. Hardell L, Carlberg M, Hansson Mild K. Pooled analysis of case-control studies on malignant brain tumours and the use of mobile and cordless phones including living and deceased subjects. International Journal of Oncology 2011; 38 (5): 1465-74. [PubMed Abstract].

18. Lönn S, Ahlbom A, Hall P, Feychting M. Longterm mobile phone use and brain tumor risk. American Journal of Epidemiology 2005; 161 (6): 526-35. [PubMed Abstract].

19. Aydin D, Feychting M, Schüz J, et al. Mobile phone use and brain tumors in children and adolescents: a multicenter case-control study. Journal of the National Cancer Institute 2011; 103 (16): 1264-76. [PubMed Abstract].

20. Inskip PD, Hoover RN, Devesa SS. Brain cancer incidence trends in relation to cellular telephone use in the United States. Neuro-Oncology 2010; 12 (11): 1147-51. [PubMed Abstract].

21. Little MP, Rajaraman P, Curtis RE, et al. Mobile phone use and glioma risk: comparison of epidemiological study results with incidence trends in the United States. British Medical Journal 2012; 344: e1147. [PubMed Abstract].

22. Deltour I, Johansen C, Auvinen A, et al. Time trends in brain tumor incidence rates in Denmark, Finland, Norway, and Sweden, 1974-2003. Journal of the National Cancer Institute 2009; 101 (24): 1721-4. [PubMed Abstract].

23. Deltour I, Auvinen A, Feychting M, et al. Mobile phone use and incidence of glioma in the Nordic countries 1979-2008: consistency check. Epidemiology 2012; 23 (2): 301-7. [PubMed Abstract].

24. U.S. Federal Communications Commission (2010). Wireless. Washington, D.C. Retrieved June 18, 2012.

25. U.S. Federal Communications Commission. (n.d.). FCC Encyclopedia: Specific Absorption Rate (SAR) for Cellular Telephones. Retrieved June 18, 2012.

26. Howlader N, Noone AM, Krapcho M, et al. (2012). SEER Cancer Statistics Review, 1975-2009 (Vintage 2009 populations). Bethesda, MD: National Cancer Institute. Retrieved June 18, 2012. 